Available online at www.sciencedirect.com

Science@Direct.

COMPTES RENDUS

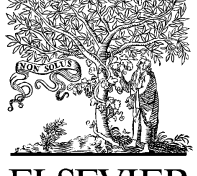

ELSEVIER

C. R. Acad. Sci. Paris, Ser. I $\bullet \bullet \bullet(\bullet \bullet \bullet) \bullet \bullet \bullet-\bullet \bullet$

Partial Differential Equations

\title{
Self-averaging radiative transfer for parabolic waves
}

\author{
Albert C. Fannjiang ${ }^{1}$ \\ Department of Mathematics, University of California at Davis, Davis, CA 95616, USA
}

Received 30 March 2005; accepted after revision 7 November 2005

Presented by Philippe G. Ciarlet

\begin{abstract}
A systematic derivations of self-averaging scaling limits of parabolic waves in terms of the Wigner distribution function is presented. The convergence of the Wigner distribution to one of the six deterministic radiative transfer equations is established. One of the main contributions of this Note is a unified framework for space-time scaling limits that lead to radiative transfer. To

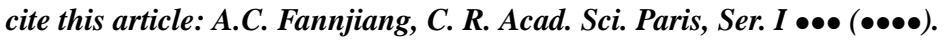

(c) 2005 Académie des sciences. Published by Elsevier SAS. All rights reserved.

\section{Résumé}

Transfert radiatif statistiquement stable pour des ondes paraboliques. On présente une méthode systématique de déduction des limites normalisatrices statistiquement stables d'ondes paraboliques en termes de la distribution de Wigner. On démontre la convergence de la distribution de Wigner vers une équation de transfert radiatif, parmi les six possibles. Une des principales contributions de cette Note réside dans un cadre unifié pour les limites normalisées en espace-temps menant au transfert radiatif.

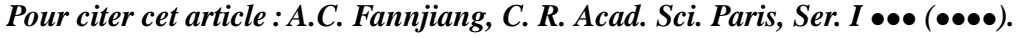

๑) 2005 Académie des sciences. Published by Elsevier SAS. All rights reserved.

\section{Version française abrégée}

On commence par l'approximation parabolique (ou paraxiale) de l'équation de Helmholtz avec longueur d'onde $2 \pi / k$ qui est décrite par l'équation de Schröginder (1) où $\Psi$ est la modulation d'amplitude, $k$ le nombre d'onde et $z$, $\mathbf{x} \in \mathbb{R}^{2}$ respectivement les coordonnées longitudinales et transverses du guide d'onde. Ensuite on normalise l' Éq. (1) par les longueurs longitudinales et transverses $L_{z}, L_{x}$ du guide d'onde, la longueur de corrélation $L_{0}$ de la fluctuation de la susceptibilité électrique et la longueur d'onde. L'équation résultant de cette normalisation est (2) où $V$ est la variation moyenne normalisée avec longueur de corrélation unitaire.

Les quatre paramètres les plus important sont les rapports $\ell_{z}=L_{z} / L_{0}, \ell_{x}=L_{x} / L_{0}$, le nombre de Fresnel $\gamma$, donné par (3), et l'amplitude de la variation $\sigma$, et toute la famille des limites normalisatrices statistiquement stables peut être

E-mail address: cafannjiang@ucdavis.edu (A.C. Fannjiang).

1 The research is supported in part by the Centennial Fellowship from American Mathematical Society and U.S. National Science Foundation grant DMS 0306659. 
décrite, quand $\gamma \rightarrow 0, \ell_{z}, \ell_{x} \rightarrow \infty$ tels que $\lim \gamma \ell_{x}<\infty$ quand $\sigma \rightarrow \infty$, de différentes manières dépendant de la relation entre $\gamma, \ell_{z}, \ell_{x}$.

Deux hypothèse principales sont faites sur la fluctuation moyenne $V:$ la première est que $V(z, \mathbf{x})$ est un processus Gaussien homogène de carré intégrable dont la densité de puissance spectrale $\Phi(\xi, \mathbf{k})$ diminue pour $|\overrightarrow{\mathbf{k}}|=\infty, \overrightarrow{\mathbf{k}}=$ $(\xi, \mathbf{k})$ avec une puissance suffisamment élevée de $|\overrightarrow{\mathbf{k}}|^{-1}$, le seconde est que le coefficient de corrélation maximal $r(t)$ défini en (4) est intégrable (5).

Un moyen utile pour analyser les ondes correspondants à des petits nombres de Fresnel est la distribution de Wigner dont la forme d'état pure est donnée par (6). La distribution de Wigner sous sa forme mixte est une combinaison convexe de ses formes d'état pure et satisfait l'équation de Wigner-Moyal (7). La distribution de Wigner préserve la majorité des informations sur la modulation d'amplitude et a une limite bien définie quand $\gamma \rightarrow 0$ (voir [13] et [10] pour plus d'éléments à ce sujet).

L'ensemble des limites normalisatrises est déduit dans deux théorèmes : le premier concerne le cas où $\lim \gamma \ell_{x}>0$ et le second concerne le cas où $\lim \gamma \ell_{x}=0$.

Théorème 0.1. Soient $\gamma \rightarrow 0, \ell_{x} \rightarrow \infty$ tels que $\lim \gamma \ell_{x}=1$. Si, de plus, $\ell_{z}, \sigma \rightarrow \infty$ sont liés par les relations ci-dessous, alors la distribution de Wigner, comme solution faible de l'équation de Wigner-Moyal, converge en probabilité à la solution de l'équation de transport $(11)$ avec $K(\mathbf{p}, \mathbf{q})$ un noyau non-négatif donné par ce qui suit :

(a) Si $\lim \sigma / \sqrt{\ell_{z}}=1, \lim \ell_{x} / \ell_{z}=0$ alors $K$ est donné par (12).

(b) $S i \lim \sigma / \sqrt{\ell_{x}}=1, \lim \ell_{x} / \ell_{z}=\infty, \lim \ell_{x} / \ell_{z}^{4 / 3}=0$ et $d \geqslant 3$ alors $K$ est donné par (13).

(c) $S i \lim \sigma / \sqrt{\ell_{z}}=1, \lim \ell_{x} / \ell_{z}=1$ alors $K$ est donné par (14).

Théorème 0.2. Soient $\gamma \rightarrow 0, \ell_{x} \rightarrow \infty$ tels que $\lim \gamma \ell_{x}=0$. Si, de plus, $\ell_{z}, \sigma \rightarrow \infty$ sont liés par les relations ci-dessous, alors la distribution de Wigner converge en probabilité, en tant que fonction faible, vers la solution de l'équation de Fokker-Planck (15) avec D étant une matrice symétrique, nonnegative-définie définie par :

(a) Si $\lim \gamma \ell_{x} \sigma / \sqrt{\ell_{z}}=1, \lim \ell_{x} / \ell_{z}=0$ alors $\mathbf{D}$ est donné par (16).

(b) Si $\lim \gamma \ell_{x} \sigma / \sqrt{\ell_{x}}=1, \lim \ell_{x} / \ell_{z}=\infty, \lim \ell_{x} / \ell_{z}^{4 / 3}=0$ et $d \geqslant 3$ alors $\mathbf{D}$ est donné par (17).

(c) Si $\lim \gamma \ell_{x} \sigma / \sqrt{\ell_{z}}=1, \lim \ell_{x} / \ell_{z}=1$ alors $\mathbf{D}$ est donné par (18).

La méthode de preuve est basée sur la méthode de fonction test perturbée $[4,12,16]$. La limitation principale des résultats ci-dessus est la condition $\lim \ell_{x} / \ell_{z}^{4 / 3}=0$ dans les Théorèmes $0.1(\mathrm{~b})$ et $0.2(\mathrm{~b})$. Ceci est dû au fait qu'on n'utilise que le premier correcteur afin que la preuve soit courte; on s'attend à ce que cette condition soit relaxée si des corrections supplémentaires sont faites dans l'analyse.

\section{Introduction}

Wave propagation in random media is an important problem for both fundamental and practical reasons. Unfortunately the full governing equations of wave propagation in random media are often extremely challenging to solve even for computers. It is thus very important to identify asymptotic regimes where simplified description can be effective and to devise efficient numerical schemes for computer simulation.

A widely used, simplified description of random waves is given by theory of radiative transfer. In this note we present a unified framework for the self-averaging regimes where a quadratic functional of the amplitude modulation, called the Wigner distribution, has a deterministic limit in the form of a transport equation in the phase space. Physically speaking, the space-time scaling framework presented here is different from previous works in the literature even though it leads to similar radiative transfer equations (cf. [7]).

Besides the limiting transport equations, the self-averaging aspect of the approximation is of paramount importance. From the perspective of the quantum open system as modeled by the Schrödinger equation with a random potential, self-averaging means that if one is only interested in large-scale observables compared to the scale of fluctuations of the environment then the quantum dynamics can have a classical, probabilistic (i.e. jump or diffusion processes in momentum) description which depends only on the covariance structure of the environment, but is 
independent of the particular realization. The transition from a unitary evolution to an irreversible process is the outcome of the phase-space coarse-graining by the test functions (the large-scale observables). Our results are a rigorous demonstration of decoherence, a mechanism believed to be responsible for the emergence of the classical world from the quantum one (see [11] and references therein). The self-averaging property also finds important technological application in time reversal experiments of waves (see $[2,3,8,9]$ and references therein).

\section{Self-averaging scaling}

Our starting point is the universal parabolic wave (or Schrödinger) equation

$$
\mathrm{i} 2 k \frac{\partial}{\partial z} \Psi(z, \mathbf{x})+\Delta \Psi(z, \mathbf{x})+k^{2} \tilde{\chi}(z, \mathbf{x}) \Psi(z, \mathbf{x})=0 .
$$

Here, in the context of quantum dynamics, $z$ would be the physical time and $\mathbf{x} \in \mathbb{R}^{d}$ the physical space and in the context of parabolic (paraxial) approximation of wave beam in a dielectric medium, $z$ would be the longitudinal coordinate and $\mathbf{x} \in \mathbb{R}^{d}$ the transverse coordinates. We have adopted the latter perspective for the rest of the note and we note that even in this case $z$ is a time-like variable. Also, the wave function $\Psi(z, \mathbf{x})$ then is the amplitude modulation, $k$ is the wavenumber, $\tilde{\chi}(z, \mathbf{x})$ is the relative fluctuation of the electric susceptibility and $\Delta$ is the Laplacian w.r.t. the transverse coordinates $\mathbf{x} \in \mathbb{R}^{d}$.

Let us nondimensionalize the equation by two reference scales of observation $L_{z}$ for the longitudinal coordinate and $L_{x}$ for the transverse coordinates. We introduce the rescaled quantities $\mathbf{x} \rightarrow \mathbf{x} L_{x}, z \rightarrow z L_{z}$ and write the electric susceptibility fluctuation as

$$
\tilde{\chi}\left(z L_{z}, \mathbf{x} L_{x}\right)=2 \frac{\ell_{x}^{2}}{\ell_{z}^{2}} U(z, \mathbf{x})+\mu V\left(z \ell_{z}, \mathbf{x} \ell_{x}\right), \quad \ell_{z}=\frac{L_{z}}{L_{0}}, \ell_{x}=\frac{L_{x}}{L_{0}}
$$

where the first term is the fluctuation on the macro-scale and the second term the fluctuation on the micro-scale with $\mu$ the standard variation and $L_{0}$ its the correlation length. Note that $V$ is the normalized small-scale electric susceptibility fluctuation with unit correlation length. After substitution and rescaling we then rewrite (1) as

$$
\mathrm{i} \frac{\partial \Psi}{\partial z}+\frac{\gamma}{2} \Delta \Psi+\gamma^{-1} U(z, \mathbf{x}) \Psi+\sigma V\left(z \ell_{z}, \mathbf{x} \ell_{x}\right) \Psi=0
$$

with the dimensionless Fresnel number

$$
\gamma=\frac{L_{z}}{k L_{x}^{2}}
$$

and the dimensionless parameter $\sigma=k L_{z} \mu / 2$.

The four most important parameters involved are the ratios $\ell_{z}=L_{z} / L_{0}, \ell_{x}=L_{x} / L_{0}$, the Fresnel number $\gamma$ and the magnitude of the fluctuation $\sigma$. The entire family of self-averaging scaling limits can be roughly described as $\gamma \rightarrow 0, \ell_{z}, \ell_{x} \rightarrow \infty$ such that $\lim \gamma \ell_{x}<\infty$ while $\sigma \rightarrow \infty$ in various ways according to the relation among $\gamma, \ell_{z}, \ell_{x}$.

\subsection{Assumptions on medium fluctuation}

First we assume that $U_{z}(\mathbf{x})=U(z, \mathbf{x}) \in C\left([0, \infty) ; \mathcal{S}\left(\mathbb{R}^{d}\right)\right)$ where $\mathcal{S}\left(\mathbb{R}^{d}\right)$ is the space of Schwartz functions on $\mathbb{R}^{d}$.

Next, we assume that $V_{z}(\mathbf{x})=V(z, \mathbf{x})$ is a centered, $z$-stationary, $\mathbf{x}$-homogeneous random field admitting the spectral representation

$$
V_{z}(\mathbf{x})=\int \exp (\mathbf{i p} \cdot \mathbf{x}) \widehat{V}_{z}(\mathrm{~d} \mathbf{p})
$$

with the $z$-stationary spectral measure $\widehat{V}_{z}(\cdot)$ satisfying

$$
\mathbb{E}\left[\widehat{V}_{z}(\mathrm{~d} \mathbf{p}) \widehat{V}_{z}(\mathrm{~d} \mathbf{q})\right]=\delta(\mathbf{p}+\mathbf{q}) \Phi_{0}(\mathbf{p}) \mathrm{d} \mathbf{p} \mathrm{d} \mathbf{q} .
$$

The transverse power spectrum density is related to the full power spectrum density $\Phi(\xi, \mathbf{p})$ as $\Phi_{0}(\mathbf{p})=$ $\int \Phi(\xi, \mathbf{p}) \mathrm{d} \xi$. The power spectral density $\Phi(\overrightarrow{\mathbf{k}})$ satisfies $\Phi(\overrightarrow{\mathbf{k}})=\Phi(-\overrightarrow{\mathbf{k}}), \forall \overrightarrow{\mathbf{k}}=(\xi, \mathbf{p}) \in \mathbb{R}^{d+1}$ because the electric 
susceptibility field is assumed to be real-valued. Hence $\Phi(w, \mathbf{p})=\Phi(-w, \mathbf{p})=\Phi(w,-\mathbf{p})=\Phi(-w,-\mathbf{p})$ which is related to the detailed balance of the limiting scattering operators described below.

More specifically we make the following two assumptions.

Assumption 1. $V(z, \mathbf{x})$ is a Gaussian process with a spectral density $\Phi(\overrightarrow{\mathbf{k}}), \overrightarrow{\mathbf{k}}=(\xi, \mathbf{p}) \in \mathbb{R}^{d+1}$ which is uniformly bounded and decays at $|\overrightarrow{\mathbf{k}}|=\infty$ with sufficiently high power of $|\overrightarrow{\mathbf{k}}|^{-1}$.

We note that the assumption of Gaussianity is not essential and is made here to simplify the presentation.

Let $\mathcal{F}_{z}$ and $\mathcal{F}_{z}^{+}$be the sigma-algebras generated by $\left\{V_{s}: \forall s \leqslant z\right\}$ and $\left\{V_{s}: \forall s \geqslant z\right\}$, respectively and let $L^{2}\left(\mathcal{F}_{z}\right)$ and $L^{2}\left(\mathcal{F}_{z}^{+}\right)$denote the square-integrable functions measurable w.r.t. to them respectively. The maximal correlation coefficient $r(t)$ is given by

$$
r(t)=\sup _{\substack{h \in L^{2}\left(\mathcal{F}_{z}\right) \\ \mathbb{E}[h]=0, \mathbb{E}\left[h^{2}\right]=1}} \sup _{\substack{g \in L^{2}\left(\mathcal{F}_{z+t}^{+}\right) \\ \mathbb{E}[g]=0, \mathbb{E}\left[g^{2}\right]=1}} \mathbb{E}[h g] .
$$

Assumption 2. The maximal correlation coefficient $r(t)$ is integrable:

$$
\int_{0}^{\infty} r(s) \mathrm{d} s<\infty
$$

Assumption 2 is very natural when the medium fluctuations decorrelate on large scales as in, for example, the turbulent atmosphere [6]. It implies certain mixing property of medium fluctuation in the time-like variable $z$ which dramatically simplifies and improves the results (presented below) over the previous ones in the literature.

\subsection{Radiative transfer equations}

We consider a family of scaling limits with $\gamma \rightarrow 0, \ell_{z}$ and $\ell_{x} \rightarrow \infty$ such that $\lim \gamma \ell_{x}<\infty$. This, of course, is not sufficient to ensure the existence of scaling limit until we specify the strength of $\sigma$.

For low-Fresnel-number waves it is convenient to use the Wigner distribution [13,10]

$$
W_{z}(\mathbf{x}, \mathbf{p})=\frac{1}{(2 \pi)^{d}} \int \mathrm{e}^{-\mathrm{i} \mathbf{p} \cdot \mathbf{y}} \Psi\left(z, \mathbf{x}+\frac{\gamma \mathbf{y}}{2}\right) \overline{\Psi\left(z, \mathbf{x}-\frac{\gamma \mathbf{y}}{2}\right)} \mathrm{d} \mathbf{y}
$$

which is always real-valued and satisfies a closed-form (Wigner-Moyal) equation

$$
\frac{\partial W_{z}}{\partial z}+\mathbf{p} \cdot \nabla W_{z}+\mathcal{U}_{z} W_{z}+\sigma \mathcal{V}_{z} W_{z}=0
$$

with

$$
\begin{aligned}
& \mathcal{U}_{z} W_{z}(\mathbf{x}, \mathbf{p})=\mathrm{i} \int \mathrm{e}^{\mathrm{i} \mathbf{q} \cdot \mathbf{x}} \gamma^{-1}\left[W_{z}(\mathbf{x}, \mathbf{p}+\gamma \mathbf{q} / 2)-W_{z}(\mathbf{x}, \mathbf{p}-\gamma \mathbf{q} / 2)\right] \widehat{U}(z, \mathrm{~d} \mathbf{q}), \\
& \mathcal{V}_{z} W_{z}(\mathbf{x}, \mathbf{p})=\mathrm{i} \int \mathrm{e}^{\mathrm{i} \mathbf{q} \cdot \mathbf{x} \ell_{x}}\left[W_{z}\left(\mathbf{x}, \mathbf{p}+\ell_{x} \gamma \mathbf{q} / 2\right)-W_{z}\left(\mathbf{x}, \mathbf{p}-\ell_{x} \gamma \mathbf{q} / 2\right)\right] \widehat{V}\left(z \ell_{z}, \mathrm{~d} \mathbf{q}\right) .
\end{aligned}
$$

Because of the linearity of (7) the mixed-state Wigner distribution which is a convex combination of the pure-state ones (6) with different $\Psi$ 's also satisfies the same equation. We consider the weak formulation

$$
\left\langle W_{z}, \theta\right\rangle-\left\langle W_{0}, \theta\right\rangle=\int_{0}^{z}\left\langle W_{s}, \mathbf{p} \cdot \nabla \theta\right\rangle \mathrm{d} s+\int_{0}^{z}\left\langle W_{s}, \mathcal{U}_{s} \theta\right\rangle \mathrm{d} s+\sigma \int_{0}^{z}\left\langle W_{s}, \mathcal{V}_{s} \theta\right\rangle \mathrm{d} s
$$

for any smooth test function $\theta$ with a compact support on the phase space where $\langle f, g\rangle=\int f(\mathbf{x}, \mathbf{p}) g(\mathbf{x}, \mathbf{p}) \mathrm{d} \mathbf{x} \mathrm{d} \mathbf{p}$ for any two real-valued functions on the phase space. 
Many useful quantities can be recovered from the Wigner distribution, which is a kind of quasi-probability density function on the phase space. Indeed one can recover from the Wigner distribution all but a constant factor, depending only on $z$, about the wave amplitude by using this property

$$
\Psi\left(z, \mathbf{x}_{1}\right) \Psi^{*}\left(z, \mathbf{x}_{2}\right)=\int W\left(z, \frac{1}{2}\left(\mathbf{x}_{1}+\mathbf{x}_{2}\right), \mathbf{q}\right) \exp \left[\mathrm{i} \gamma^{-1} \mathbf{q} \cdot\left(\mathbf{x}_{1}-\mathbf{x}_{2}\right)\right] \mathrm{d} \mathbf{q} .
$$

The family of self-averaging scaling limits can be divided into two groups depending on whether $\lim \gamma \ell_{x}$ is positive (set to one) or zero.

The following result is a reformulation and extension of those of [7]. The main differences from [7] are (i) the simplified Assumption 1, (ii) the additional macroscopic inhomogeneities represented by $U$ which introduces a nontrivial complication in constructing the perturbed test function and, most importantly, (iii) a different, unified framework for space-time scaling in terms of $\gamma, \ell_{x}, \ell_{z}$.

Theorem 2.1. Let $\gamma \rightarrow 0, \ell_{x} \rightarrow \infty$ such that $\lim \gamma \ell_{x}=1$.

If in addition $\ell_{z}, \sigma \rightarrow \infty$ in one of the following ways the Wigner distribution, as a weak solution of the WignerMoyal equation, converges in probability in the space $C\left([0, \infty) ; L^{2}\left(\mathbb{R}^{2 d}\right)\right)$ to the solution of the transport equation

$$
\frac{\partial}{\partial z} \bar{W}_{z}(\mathbf{x}, \mathbf{p})+\mathbf{p} \cdot \nabla \bar{W}_{z}(\mathbf{x}, \mathbf{p})-\nabla_{\mathbf{x}} U \cdot \nabla_{\mathbf{p}} \bar{W}_{z}(\mathbf{x}, \mathbf{p})=2 \pi \int K(\mathbf{p}, \mathbf{q})\left[\bar{W}_{z}(\mathbf{x}, \mathbf{q})-\bar{W}_{z}(\mathbf{x}, \mathbf{p})\right] \mathrm{d} \mathbf{q}
$$

where $K(\mathbf{p}, \mathbf{q})$ is a nonnegative kernel given as follows.

(a) If $\lim \sigma / \sqrt{\ell_{z}}=1, \lim \ell_{x} / \ell_{z}=0$ then $K(\mathbf{p}, \mathbf{q})=\Phi(0, \mathbf{q}-\mathbf{p})$;

(b) If $\lim \sigma / \sqrt{\ell_{x}}=1, \lim \ell_{x} / \ell_{z}=\infty, \lim \ell_{x} / \ell_{z}^{4 / 3}=0$ and $d \geqslant 3$ then

$$
K(\mathbf{p}, \mathbf{q})=\delta\left(\frac{|\mathbf{q}|^{2}-|\mathbf{p}|^{2}}{2}\right)\left[\int \Phi(w, \mathbf{q}-\mathbf{p}) \mathrm{d} w\right] ;
$$

(c) If $\lim \sigma / \sqrt{\ell_{z}}=1, \lim \ell_{x} / \ell_{z}=1$ then $K(\mathbf{p}, \mathbf{q})=\Phi\left(\frac{|\mathbf{q}|^{2}-|\mathbf{p}|^{2}}{2}, \mathbf{q}-\mathbf{p}\right)$.

Theorem 2.2. Let $\gamma \rightarrow 0, \ell_{x} \rightarrow \infty$ such that $\lim \gamma \ell_{x}=1$. If in addition $\ell_{z}, \sigma \rightarrow \infty$ in one of the following ways the Wigner distribution converges in probability in the space $C\left([0, \infty) ; L^{2}\left(\mathbb{R}^{2 d}\right)\right)$ as a weak function to the solution of the transport equation

$$
\frac{\partial}{\partial z} \bar{W}_{z}+\mathbf{p} \cdot \nabla \bar{W}_{z}-\nabla_{\mathbf{x}} U \cdot \nabla_{\mathbf{p}} \bar{W}_{z}=\nabla_{\mathbf{p}} \cdot \mathbf{D} \nabla_{\mathbf{p}} \bar{W}_{z}
$$

where $\mathbf{D}$ is a symmetric, nonnegative-definite matrix given as follows.

(a) If $\lim \gamma \ell_{x} \sigma / \sqrt{\ell_{z}}=1, \lim \ell_{x} / \ell_{z}=0$ then $\mathbf{D}=\pi \int \Phi(0, \mathbf{q}) \mathbf{q} \otimes \mathbf{q} \mathrm{d} \mathbf{q}$;

(b) If $\lim \gamma \ell_{x} \sigma / \sqrt{\ell_{x}}=1, \lim \ell_{x} / \ell_{z}=\infty, \lim \ell_{x} / \ell_{z}^{4 / 3}=0$ and $d \geqslant 3$ then

$$
\mathbf{D}(\mathbf{p})=\pi|\mathbf{p}|^{-1} \int_{\mathbf{p} \cdot \mathbf{p}_{\perp}=0} \int \Phi\left(w, \mathbf{p}_{\perp}\right) \mathrm{d} w \mathbf{p}_{\perp} \otimes \mathbf{p}_{\perp} \mathrm{d} \mathbf{p}_{\perp} ;
$$

(c) If $\lim \gamma \ell_{x} \sigma / \sqrt{\ell_{z}}=1, \lim \ell_{x} / \ell_{z}=1$ then $\mathbf{D}(\mathbf{p})=\pi \int \Phi(\mathbf{p} \cdot \mathbf{q}, \mathbf{q}) \mathbf{q} \otimes \mathbf{q} \mathrm{d} \mathbf{q}$.

Eq. (15) with (16), (17) and (18) is the geometrical optics limit of Eq. (11) with (12), (13) and (14), respectively.

The standard radiative transfer scaling is the one that leads to Eq. (11) with the kernel (14). This has been established previously in [1] for a special Markovian fluctuation $V_{z}$ in the absence of large-scale inhomogeneities $U=0$. Eq. (11) with (12) is closely related to the mean Wigner distribution in the white-noise model proved in [6], while Eq. (11) with (13) is closely related to the $z$-independent model [15,5] where the convergence of the mean field $\mathbb{E}\left[W_{z}\right]$, in small and large times respectively, is proved (see also [14]). In the $z$-independent model the convergence of the full field is not known. 
The main idea of the proof is to consider the infinitesimal operator (pseudo-generator) $\mathcal{A}$ for the process (10) and use perturbed test functions $[4,12,16]$. For a function on the probability space $C\left([0, \infty) ; L_{w}^{2}\left(\mathbb{R}^{2 d}\right)\right)$ of the form $f_{z} \equiv f\left(\left\langle W_{z}, \theta\right\rangle\right)$ with $f \in C^{\infty}(\mathbb{R}) \mathcal{A} f_{z}$ is given by

$$
\mathcal{A} f_{z}=f_{z}^{\prime}\left[\left\langle W_{z}, \mathbf{p} \cdot \nabla \theta\right\rangle+\left\langle W_{z}, \mathcal{U}_{z} \theta\right\rangle+\sigma\left\langle W_{z}, \mathcal{V}_{z} \theta\right\rangle\right]
$$

For us, the most important property of $\mathcal{A}$ is that

$$
f_{z}-\int_{0}^{z} \mathcal{A} f_{s} \mathrm{~d} s \text { is a } \mathcal{F}_{z} \text {-martingale. }
$$

We then construct a perturbed test function $f_{z}^{\gamma}$ which approaches $f_{z}$ as $\gamma \rightarrow 0$ in the suitable sense. Using the perturbed test function we first prove tightness and obtain a limit $\bar{W}_{z}$. For $f_{z}^{\gamma}$ we show that the quadratic variation of the martingale vanishes in the limit and $\mathcal{A} f_{z}^{\gamma}$ converges to $\overline{\mathcal{A}} f_{z}$ given by

$$
\overline{\mathcal{A}} f(z) \equiv f_{s}^{\prime}\left[\left\langle\bar{W}_{z}, \mathbf{p} \cdot \nabla_{\mathbf{x}} \theta\right\rangle+A_{0}\left(\bar{W}_{z}\right)+A_{1}\left(\bar{W}_{z}, \theta\right)\right]
$$

with $A_{0}=-\left\langle W_{z}, \nabla_{\mathbf{x}} U \cdot \nabla_{\mathbf{p}} \theta\right\rangle$ and $A_{1}$ being the bilinear form associated with the scattering operator on the right-hand side of Eq. (11) or (15). Therefore we conclude that the limiting process is deterministic.

The main restriction of the above results lies in the condition $\lim \ell_{x} / \ell_{z}^{4 / 3}=0$ in the case of Theorems 2.1(b) and 2.2(b). This is because we use only the first corrector for the brevity of argument; we expect the condition to be relaxed if more correctors are used in the analysis.

\section{Acknowledgement}

I thank Dr. Boumediene Hamzi for translating the English manuscript into French and Dr. Daniel Coutand for reading the manuscript.

\section{References}

[1] G. Bal, G. Papanicolaou, L. Ryzhik, Stochastic Dynamics 2 (2002) 507-531.

[2] C. Bardos, M. Fink, Asymptotic Anal. 29 (2002) 157-182.

[3] P. Blomgren, G. Papanicolaou, H. Zhao, J. Acoust. Soc. Amer. 111 (2002) 230.

[4] R. Bouc, E. Pardoux, Stochastic Anal. Appl. 2 (1984) 369-422.

[5] L. Erdös, H.T. Yau, Comm. Pure Appl. Math. 53 (2000) 667-735.

[6] A. Fannjiang, Comm. Math. Phys. 254 (2) (2005) 289-322.

[7] A. Fannjiang, Arch. Rational Mech. Anal. 175 (3) (2005) 343-387.

[8] A. Fannjiang, physics/0509158.

[9] M. Fink, D. Cassereau, A. Derode, C. Prada, P. Roux, M. Tanter, J.L. Thomas, F. Wu, Rep. Progr. Phys. 63 (2000) $1933-1995$.

[10] P. Gerard, P.A. Markowich, N.J. Mauser, F. Poupaud, Comm. Pure Appl. Math. L (1997) 323-379.

[11] E. Joos, H.D. Zeh, I. Stamatescu, Decoherence and the Appearance of a Classical World in Quantum Theory, Springer-Verlag, New York, 2003.

[12] H.J. Kushner, Approximation and Weak Convergence Methods for Random Processes, with Applications to Stochastic Systems Theory, The MIT Press, Cambridge, MA, 1984.

[13] P.-L. Lions, P. Paul, Rev. Mat. Iberoamericana 9 (1993) 553-618.

[14] F. Poupaud, A. Vasseur, Math. Pure Appl. 82 (2003) 711-748.

[15] H. Spohn, J. Statist Phys. 17 (1977) 385-412.

[16] L. Tartar, Cours Peccot, College de France, 1977-1978. 